\title{
A novel multi-image cryptosystem based on weighted plain images and using combined chaotic maps
}

Ahmad Pourjabbar Kari ( $\sim$ a.pourjabar@gmail.com )

Islamic Azad University Tehran North Branch https://orcid.org/0000-0001-5303-3453

Ahmad Habibizad Navin

Islamic Azad University Tabriz Branch

Amir Massoud Bidgoli

Islamic Azad University Tehran North Branch

Mirkamal Mirnia

Tabriz University: University of Tabriz

Original Research Paper

Keywords: Multimedia security, Image encryption, Information entropy, Noise attack, Chaotic maps, Henon map

Posted Date: February 3rd, 2021

DOl: https://doi.org/10.21203/rs.3.rs-164388/v1

License: (9) This work is licensed under a Creative Commons Attribution 4.0 International License. Read Full License

Version of Record: A version of this preprint was published at Multimedia Systems on March 13th, 2021. See the published version at https://doi.org/10.1007/s00530-021-00772-y. 


\section{Abstract}

This paper introduces a new multi-image cryptosystem based on modified Henon map and nonlinear combination of chaotic seed maps. Based on the degree of correlation between the adjacent pixels of the plain image, a unique weight is assigned to the plain image. First, the coordinates of plain images are disrupted by modified Henon map as confusion phase. In the first step of diffusion phase, the pixels content of images are changed separately by XOR operation between confused images and matrices with suitable nonlinear combination of seed maps sequences. These combination of seed maps are selected depending on the weight of plain images as well as bifurcation properties of mentioned chaotic maps. After concatenating the matrices obtained from the first step of diffusion phase, the bitwise XOR operation is applied between newly developed matrix and the other produced matrix from the chaotic sequences of the Logistic-Tent-Sine hybrid system, as second step of diffusion phase. The encrypted image is obtained after applying shift and exchange operations. The results of the implementation using graphs and histograms show that the proposed scheme, compared to some existing methods, can effectively resist common attacks and can be used as a secure method for encrypting digital images.

\section{Full Text}

This preprint is available for download as a PDF.

\section{Figures}

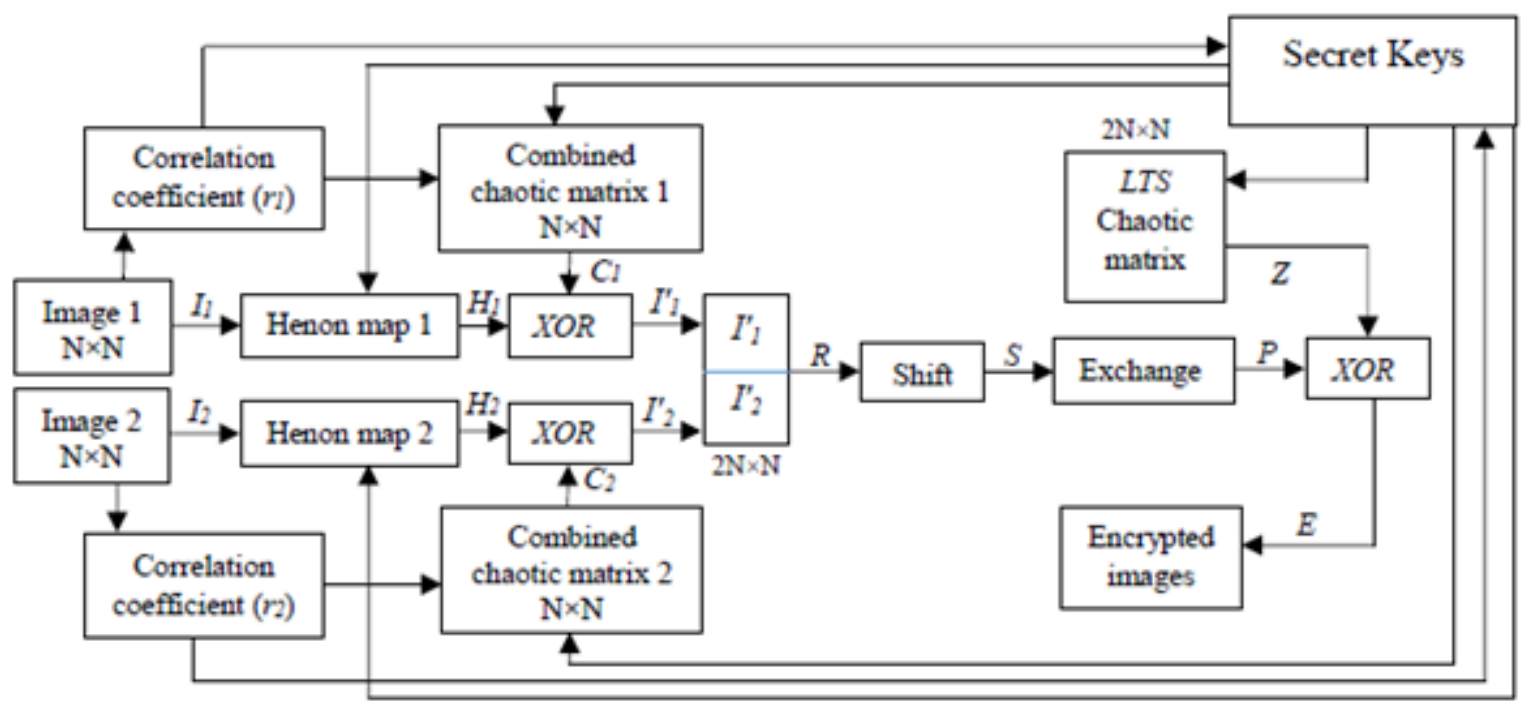

\section{Figure 1}

Block diagram of the proposed method 

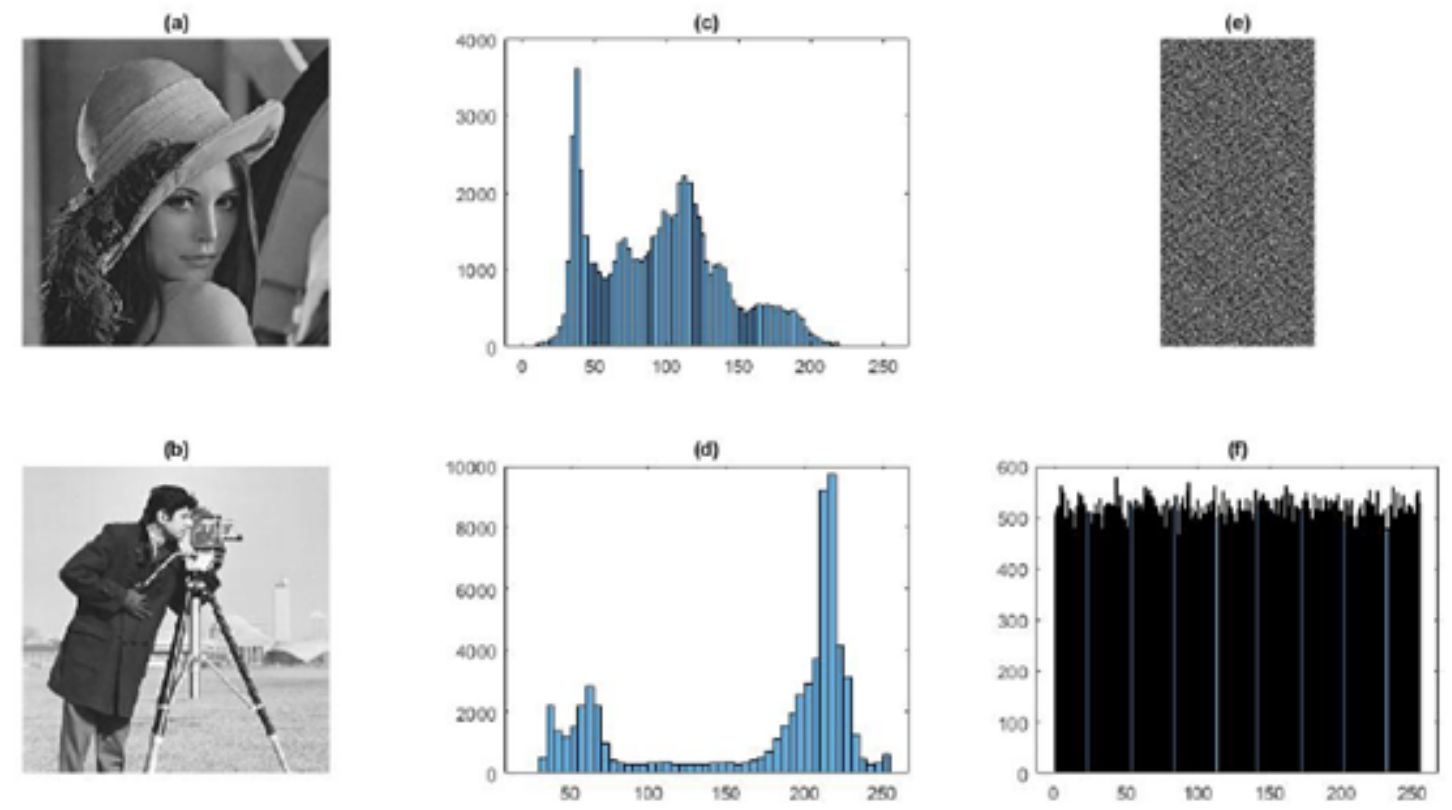

\section{Figure 2}

(a) standard 256×256 "Lena" plain image, (b) standard 256×256 "Camera man" plain image, second column (c), (d) are plain images with non-uniform histograms respectively, (e) is the $512 \times 256$ two plain image single encrypted image, (f) is the uniform histogram of double encrypted images
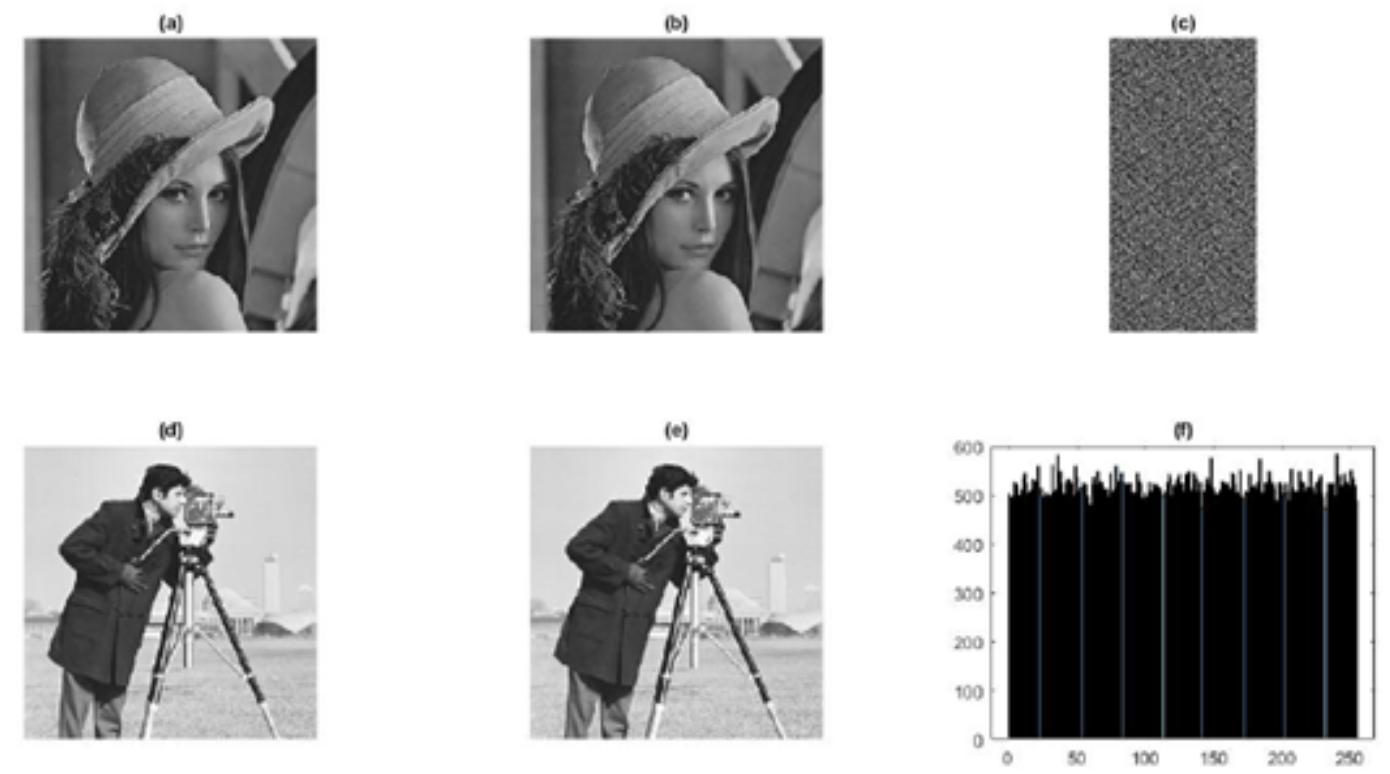

\section{Figure 3}

First column (a), (d) are two $256 \times 256$ plain images, second column (b), (e) are decrypted images respectively, (c) is the $512 \times 256$ two plain image single encrypted image, (f) is the uniform histogram of double encrypted images 

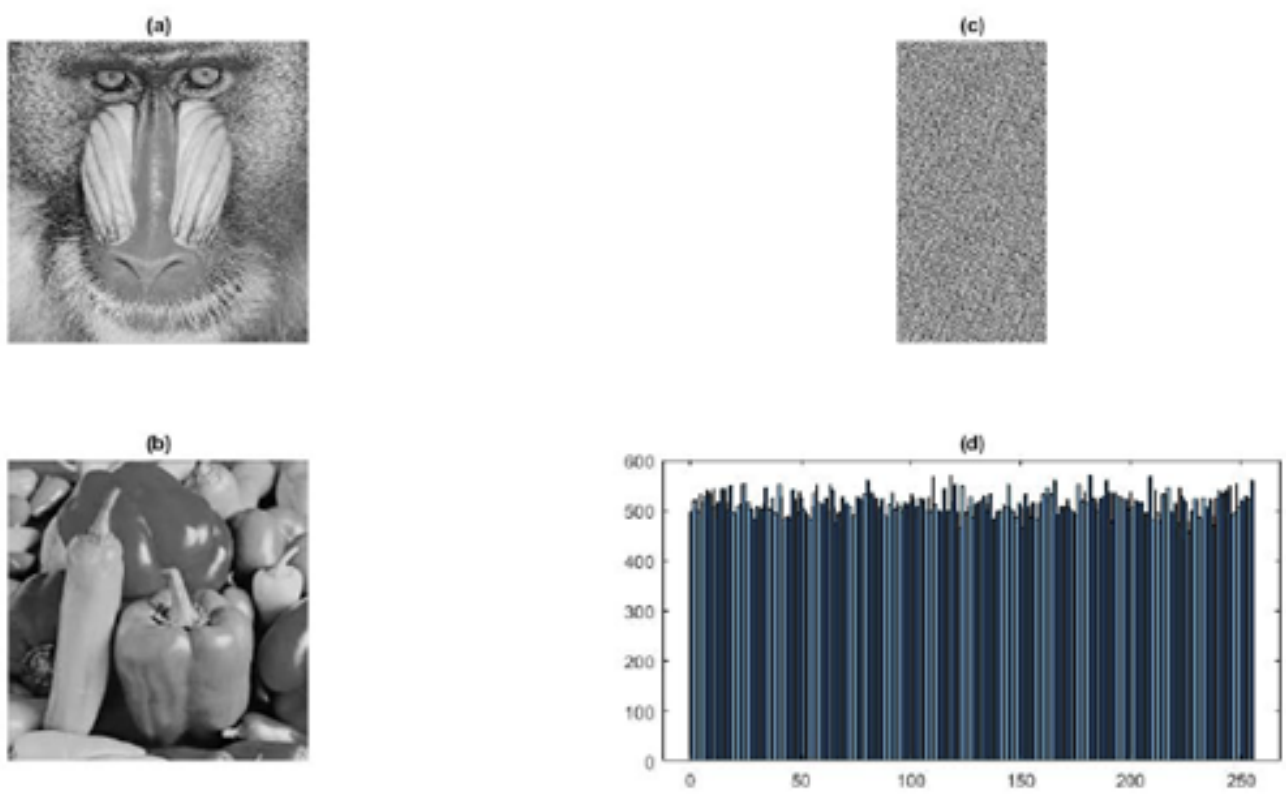

\section{Figure 4}

(a) standard 256×256 "Baboon" plain image, (b) standard 256×256 "Peppers" plain image, (c) is the $512 \times 256$ two plain image single encrypted image, (d) is the uniform histogram of encrypted images
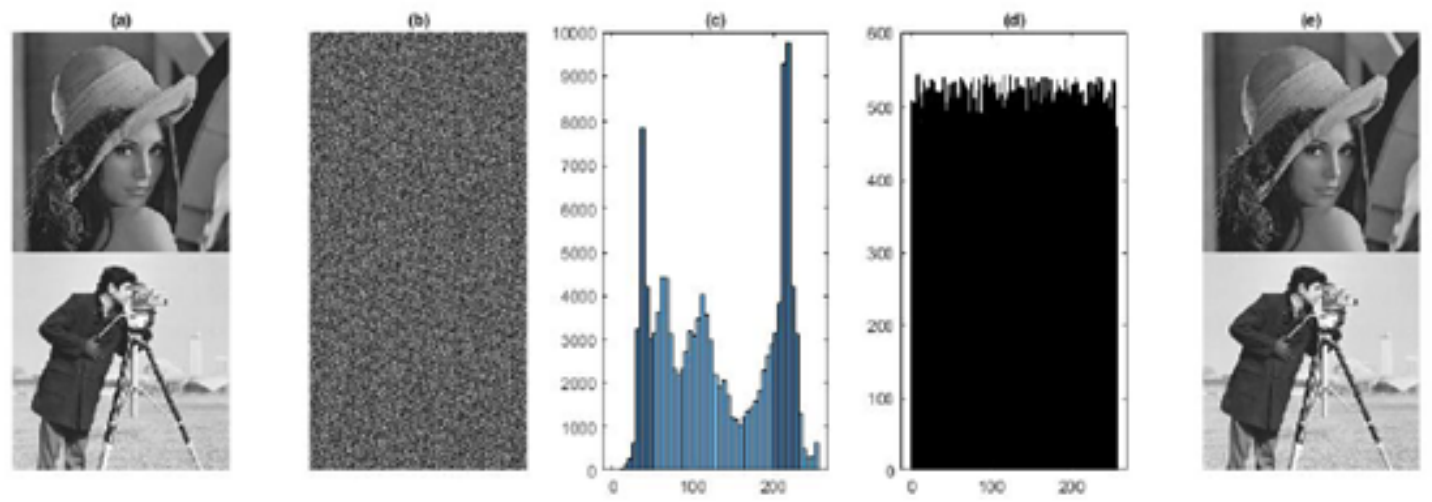

\section{Figure 5}

(a) is $512 \times 256$ merged two $256 \times 256$ plain images, (b) is the $512 \times 256$ two plain image single encrypted image, (c) is the histogram of two concatenated plain images, (d) is the uniform histogram of encrypted images, (e) is the decrypted image 

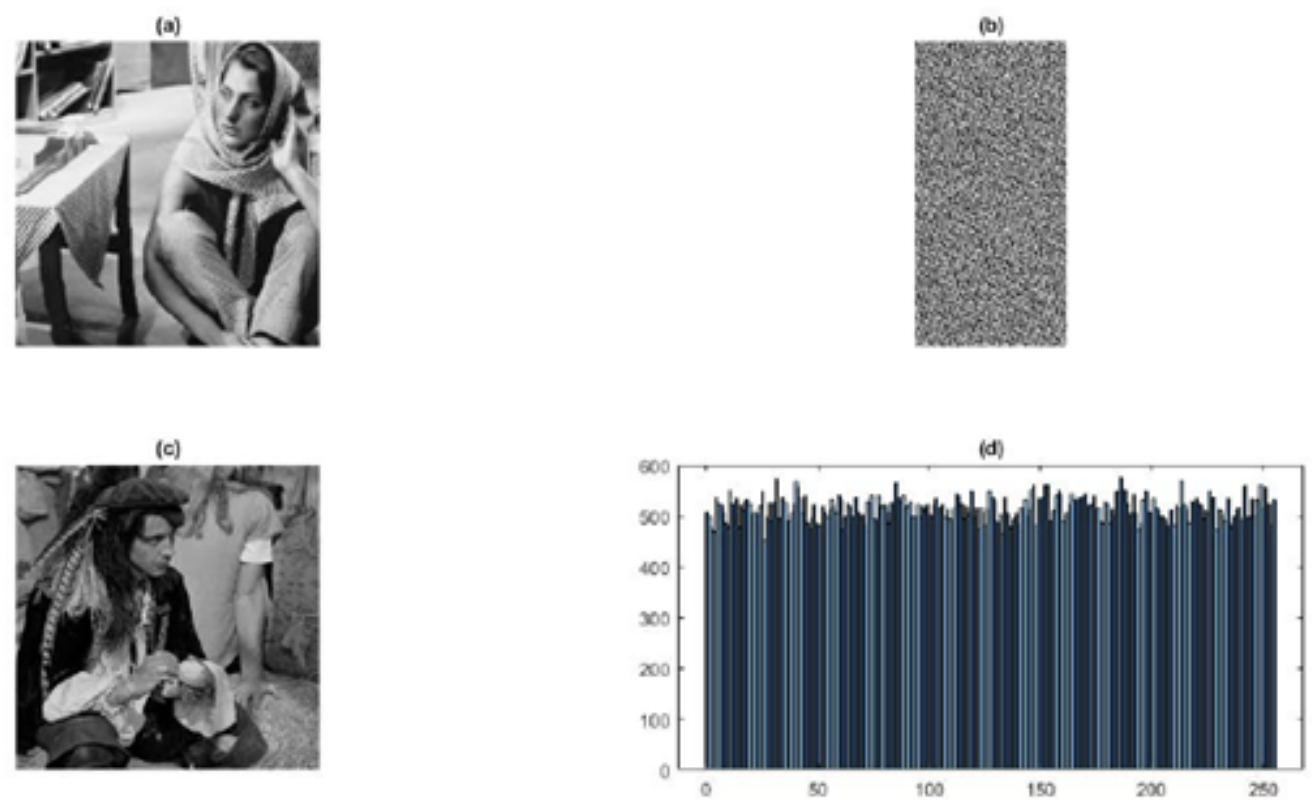

\section{Figure 6}

(a) standard 256×256 "Female" plain image, (c) standard "Male" 256×256 plain image, (b) is the $512 \times 256$ two plain image single encrypted image, (d) is the uniform histogram of encrypted images
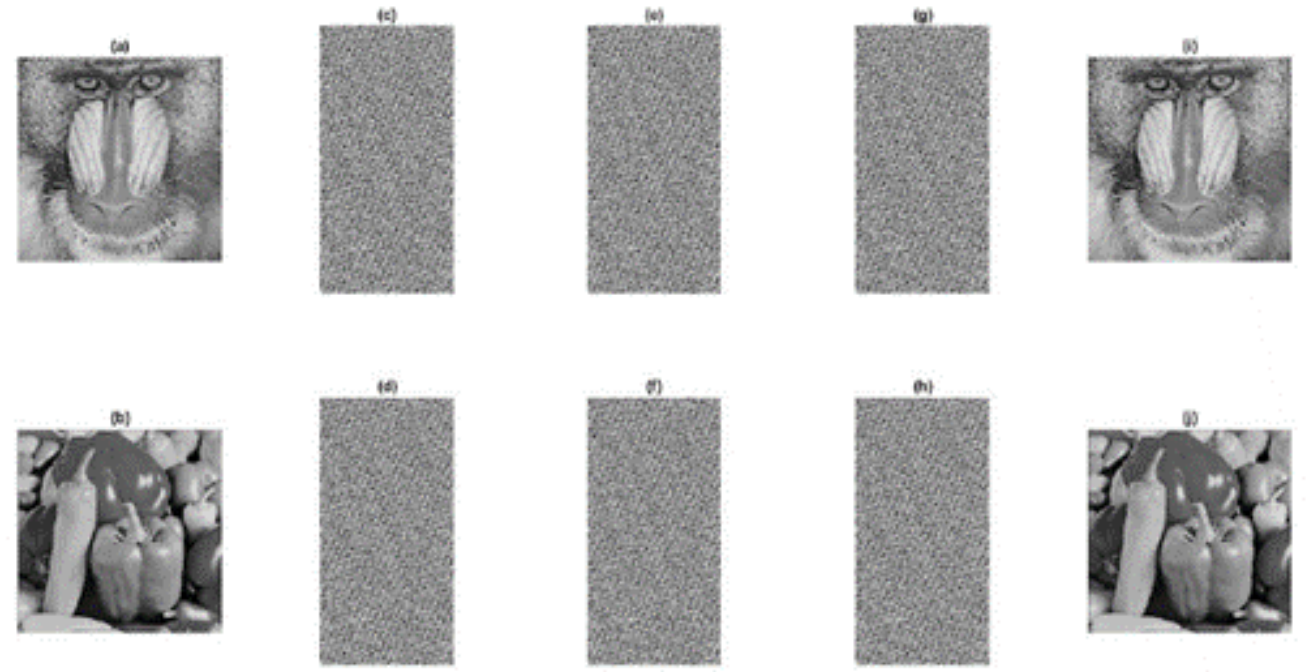

\section{Figure 7}

(a) and (b) are $256 \times 256$ plain images, (c) is the $512 \times 256$ encrypted image of two plain images with secret key Ke1 $=0.78$, (d) is the $512 \times 256$ encrypted image of two plain images with secret key Ke2 $=0.83$, (e) is the $512 \times 256$ decrypted image of two plain images with secret key Ke3 $=0.78+10-14$, $(\mathrm{f})$ is the $512 \times 256$ decrypted image of two plain images with secret key Ke4=0.83+10-14, $(\mathrm{g})$ is the $512 \times 256$ decrypted image of two plain images with secret key Ke5=0.93, (h) is the $512 \times 256$ decrypted image of 
two plain images with secret key Ke4=0.63, (i) is the decrypted image of plain image (a) with correct secret key Ke1 $=0.78,(j)$ is the decrypted image of plain image (b) with correct secret key Ke2 $=0.83$

(a)

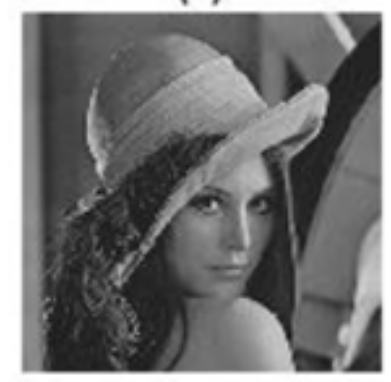

(d)

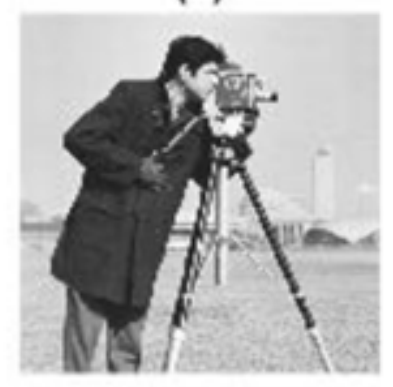

(b)

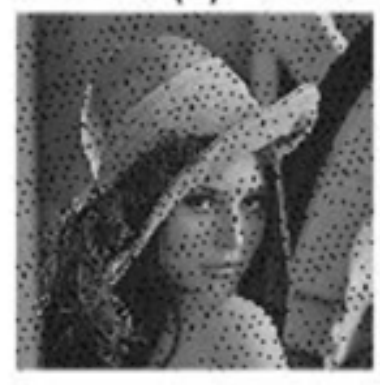

(c)

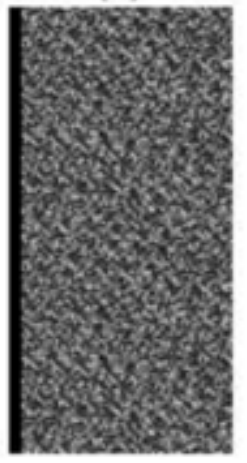

(f)

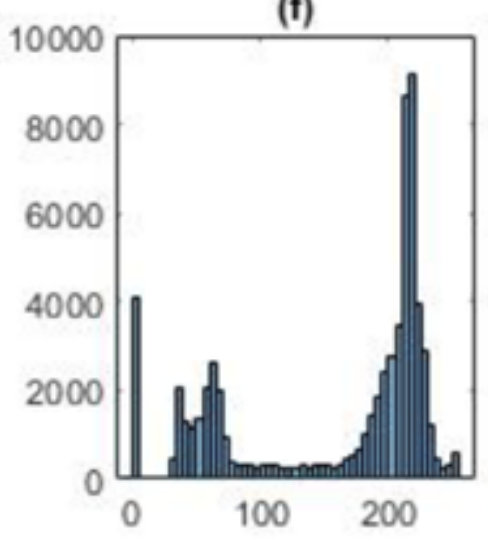

Figure 8

(a) and (d) are $256 \times 256$ plain images, (b) is the decrypted image for (a) after 1/16 cropping attack in one round, (e) is the decrypted image for (d) after 116 cropping attack in one round, (c) is the $512 \times 256$ two plain image single encrypted image after $1 / 16$ cropping attack, $(\mathrm{f})$ is the histogram of $(\mathrm{e})$ 
(a)

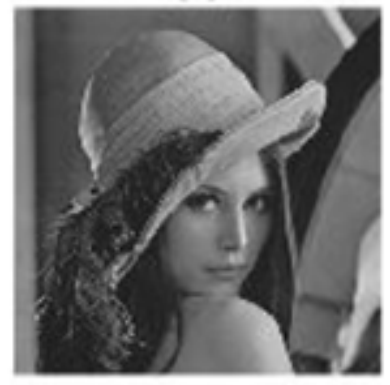

(d)

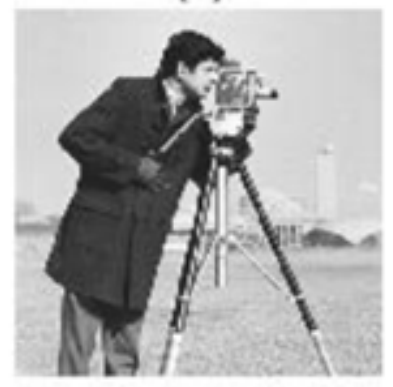

(b)

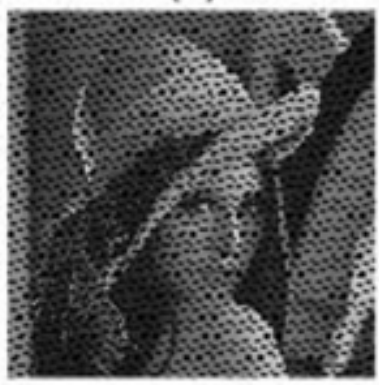

(e)

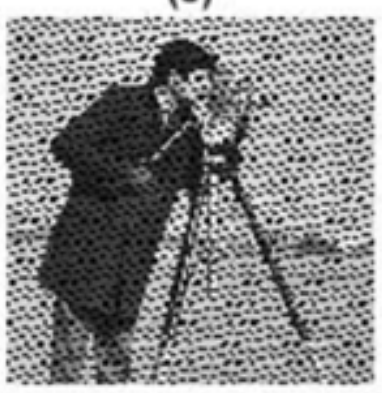

(c)
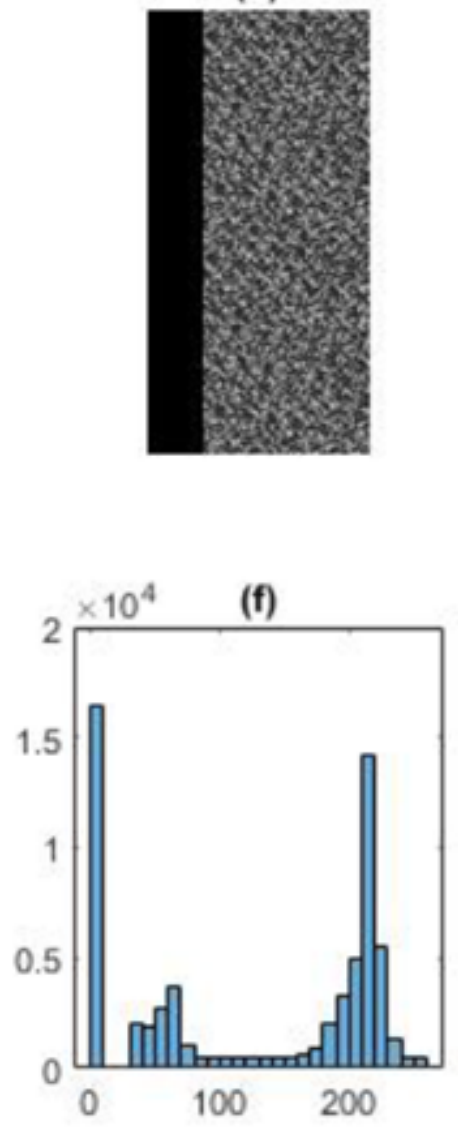

Figure 9

a) and (d) are $256 \times 256$ plain images, (b) is the decrypted image for (a) after $1 / 4$ cropping attack, (e) is the decrypted image for (d) after $1 / 4$ cropping attack, (c) is the $512 \times 256$ two plain image single encrypted image after $1 / 4$ cropping attack, $(\mathrm{f})$ is the histogram of $(\mathrm{e})$ 
(a)

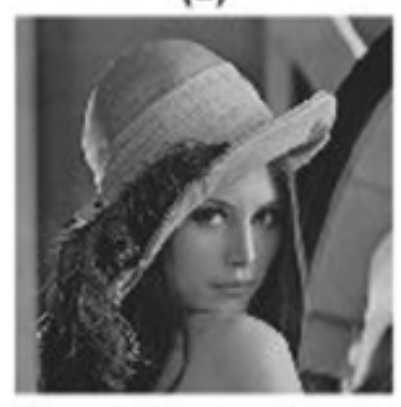

(d)

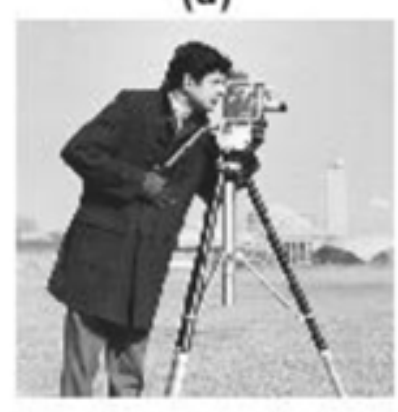

(b)

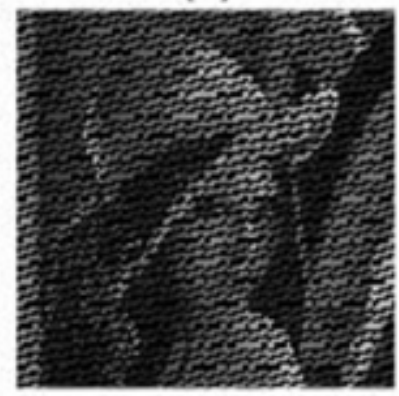

(e)

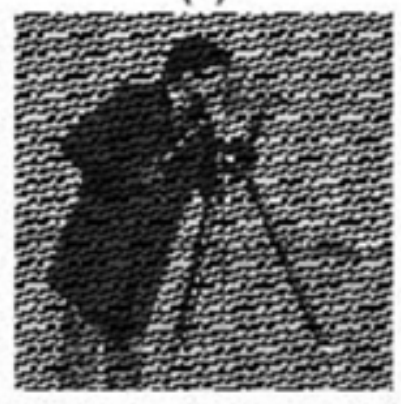

(c)
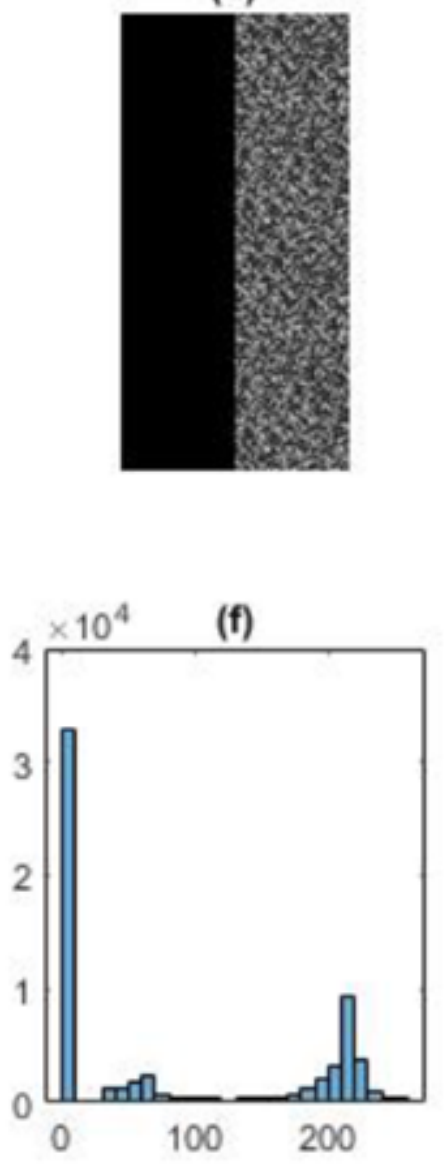

Figure 10

(a) and (d) are $256 \times 256$ plain images, (b) is the decrypted image for (a) after $1 / 2$ cropping attack, (e) is the decrypted image for (d) after $1 / 2$ cropping attack, (c) is the $512 \times 256$ two plain image single encrypted image after $1 / 2$ cropping attack, $(f)$ is the histogram of (e) 
(a)

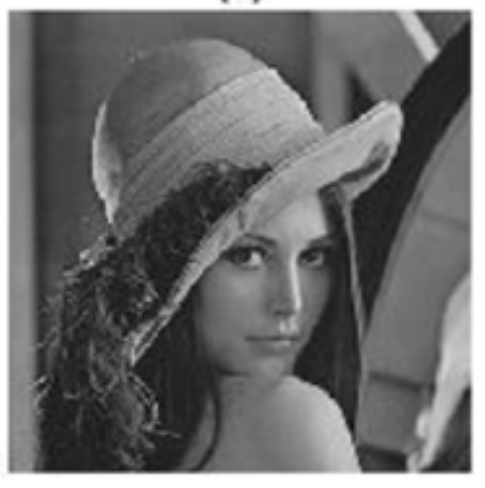

(c)

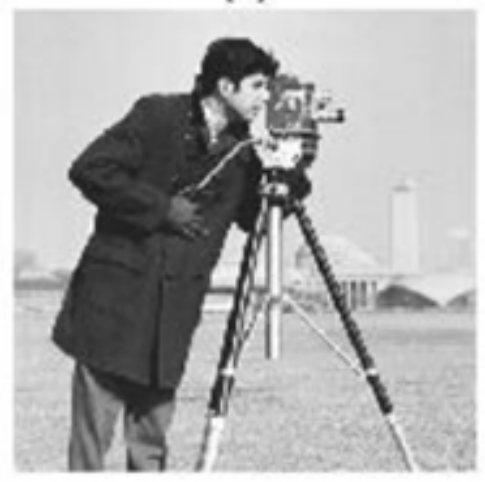

(b)

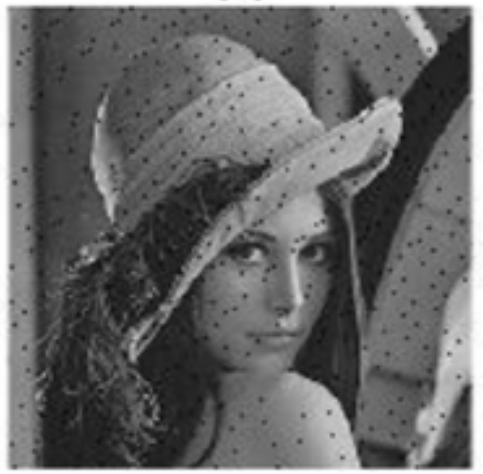

(d)

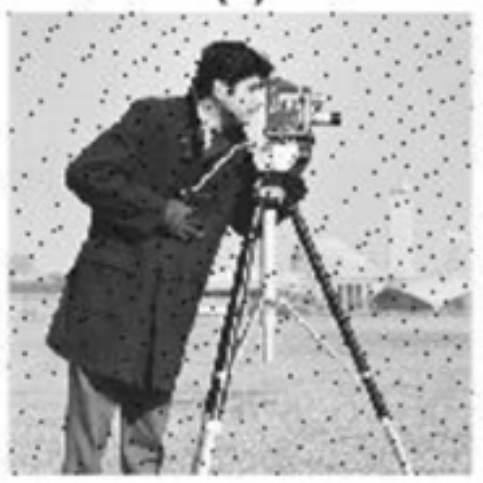

\section{Figure 11}

a) and (c) are $256 \times 256$ plain images, (b) is the decrypted image for (a) after $5 \%$ salt and pepper attack, (d) is the decrypted image for (c) after $5 \%$ salt and pepper attack 


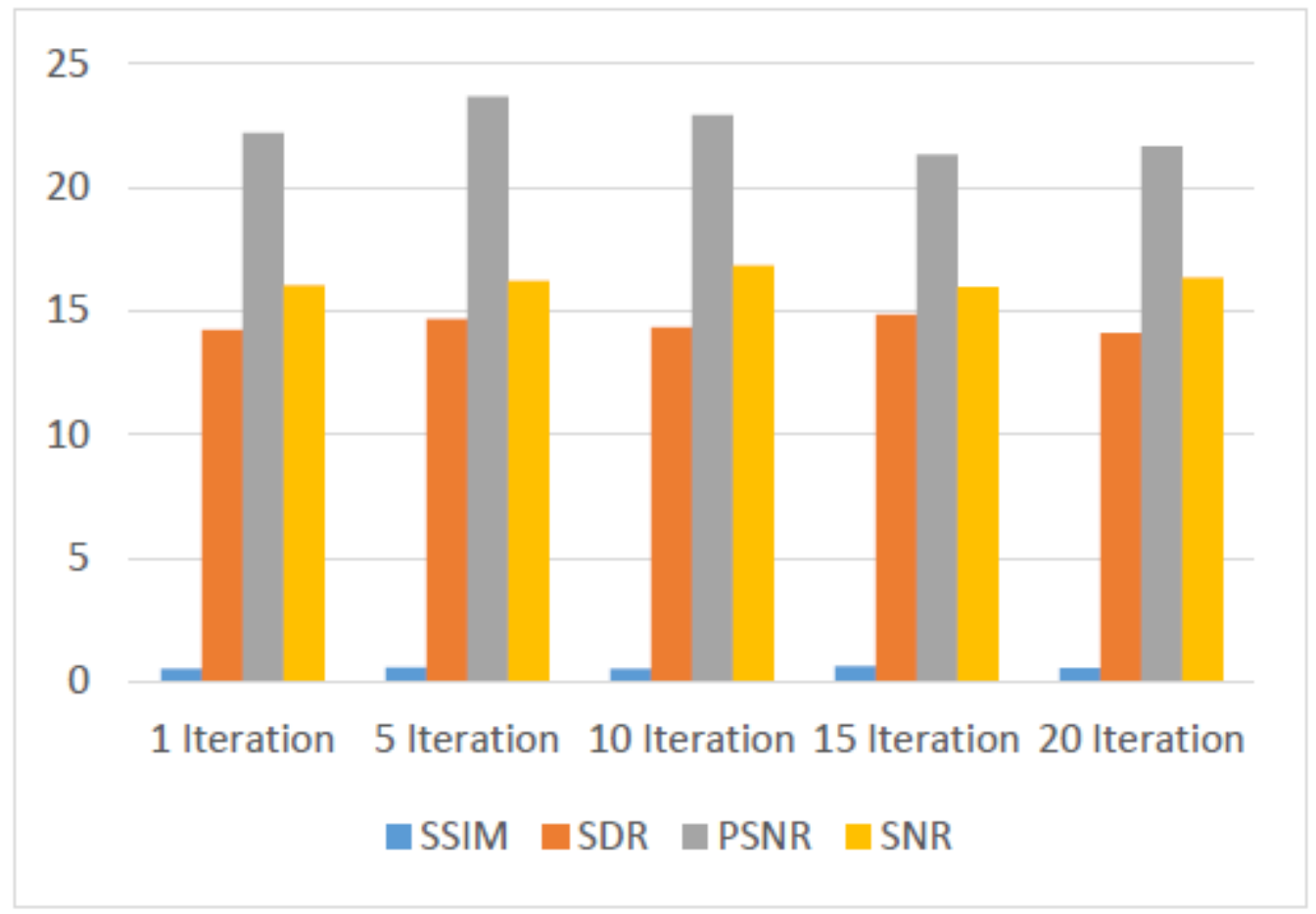

Figure 12

Evaluating parameters for various iterations, and 1/16 cropping attack

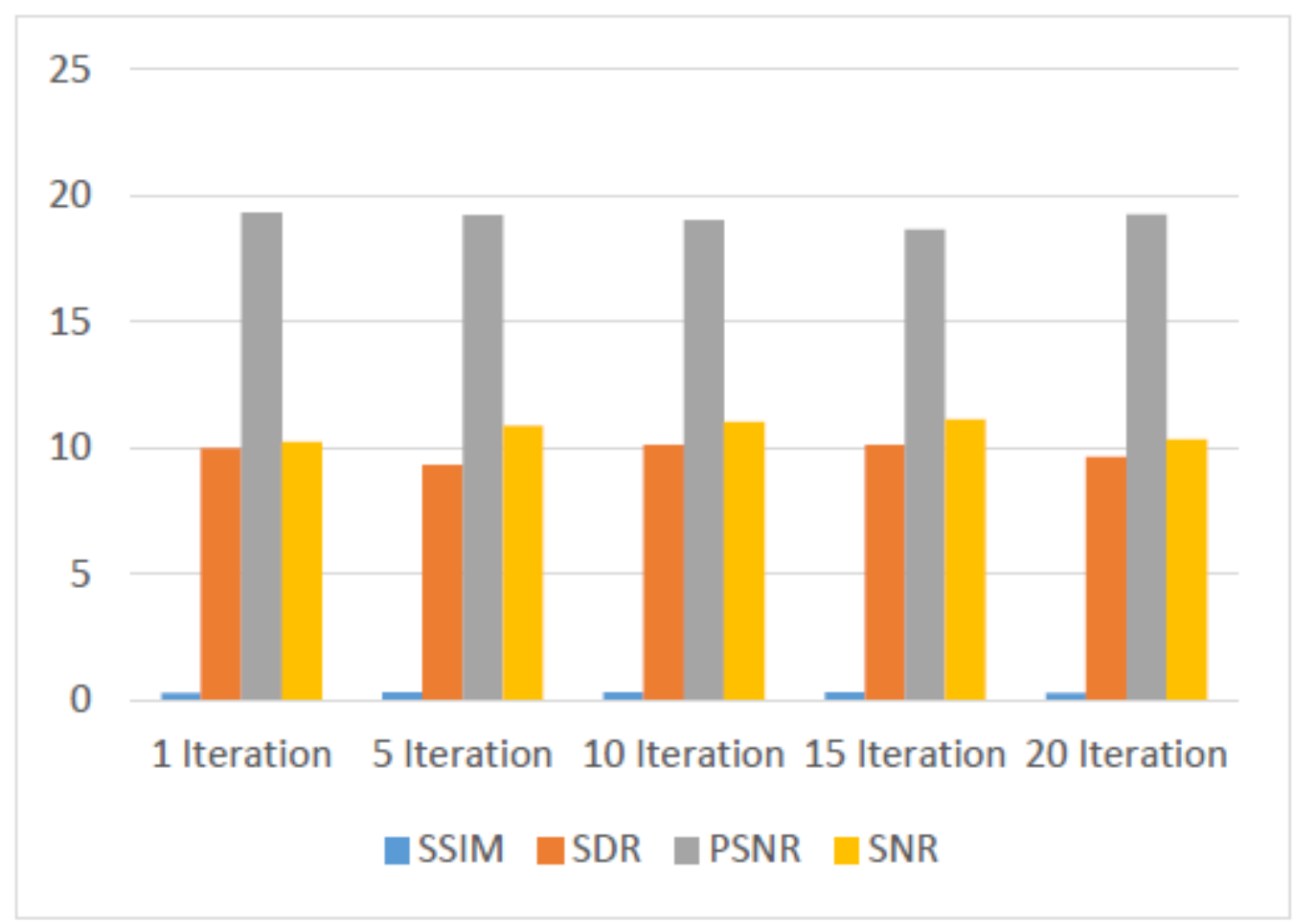

Figure 13 
Evaluating parameters for various iterations, and 1/4 cropping attack

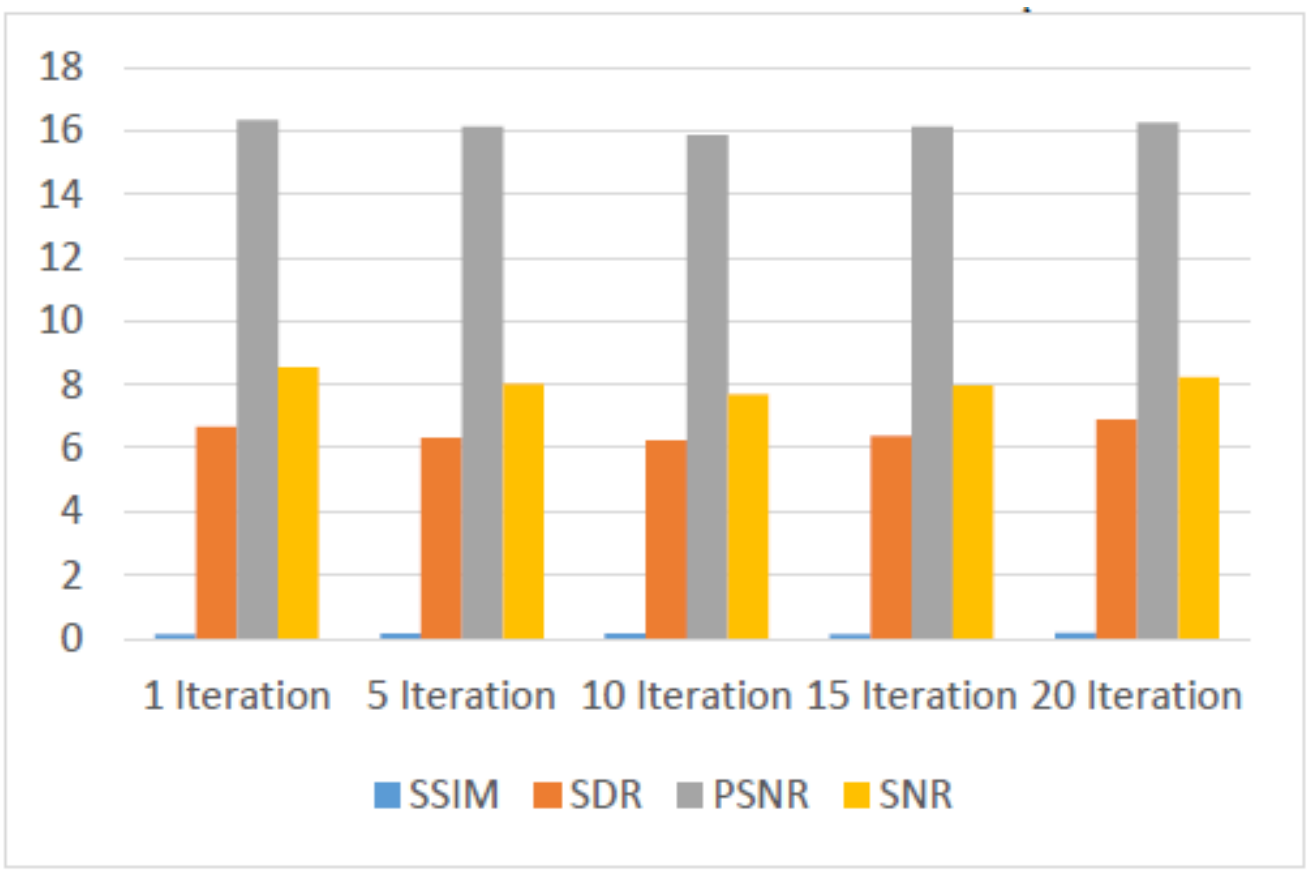

Figure 14

Evaluating parameters for various iterations, and 1/2 cropping attack 